REVISIÓN DE LITERATURA

\title{
Metanogénesis microbiana en animales poligástricos
}

Daniel Javier Bonilla-Sandí A1, Lucía Noboa-Jiménez A2, Victoria Portuguez-Molina A3E, Fernando QuintoUreña ${ }^{A 4}$, Joel José Rojas-Gutiérrez ${ }^{\text {AS }}$

\section{RESUMEN}

La metanogénesis ruminal consiste en reacciones que sintetizan metano a partir de moléculas orgánicas y son realizadas por arqueas metanogénicas en el rumen. La metanogénesis microbiana en animales rumiantes trae consigo grandes repercusiones tanto en el metabolismo y productividad comercial de los mismos como en la estabilidad del medio ambiente en general. Uno de los principales factores que pueden afectar es la dieta del rumiante, así como su edad y la especie. Las estrategias de mitigación están orientadas a la disminución de la producción de gases de efecto invernadero (GEI) o el aumento de los mecanismos de captura de compuestos formadores de GEI. Pueden clasificarse en tres categorías: manipulación alimentaria y dietética, manipulación animal y manipulación microbiana. Estas influyen en la alteración del ecosistema ruminal y la tasa de metanogénesis. La cuantificación del metano producido por rumiantes permite orientar la erradicación de factores que favorecen la metanogénesis.

Palabras clave: Metanógenos, alimentación, rumen, mitigación, defaunación.

\section{ABSTRACT}

Microbial Metanogenesis in poligastric animals. Ruminal methanogenesis consists in reactions that synthesize methane from organic molecules and are performed by methanogenic archeas in the rumen. Microbial methanogenesis in ruminant animals has repercussions both in their metabolism and commercial productivity, and in the stability of the environment.One of the most important factors is the rumian diet,its age and the species. Mitigation strategies are oriented to reduce greenhouse gas (GHG) production or to produce mechanisms that captures GHG-forming compounds. They can be classified into three categories: food and dietary manipulation, animal manipulation and microbial manipulation. These influence the alteration of the rumen ecosystem and the rate of methanogenesis. Quantification of methane produced by ruminants leads the eradication of factors that stimulate methanogenesis.

Key words: Methanogens, feeding, rumen, mitigation, defaunation.

\footnotetext{
A Instituto Tecnológico de Costa Rica, estudiante de la carrera Ingeniería en Biotecnología. Cartago, Costa Rica.

1'dabosa08@hotmail.com, 2 lucianoboa99@gmail.com, ${ }^{3}{ }^{\circledR}$ Autora para correspondencia: vicpm08@gmail.com,

${ }^{4}$ quintofernando99@gmail.com, ${ }^{5}$ jorogu2000@gmail.com

Recibido: 19 noviembre $2019 \quad$ Aceptado: 9 junio 2020

Esta obra está bajo licencia internacional Creative Commons Reconocimiento-NoComercial-SinObrasDerivadas 4.0. 


\section{INTRODUCCIÓN}

El rumen realiza procesos de fermentación, como menciona Galindo et al. (2017), este órgano representa la mayoría del volumen del sistema digestivo, y en sus reacciones se libera una cantidad importante de gases. Entre los productos de las reacciones se encuentra el metano liberado propiamente por la metanogénesis. La microbiota del rumen está constituida por hongos, arqueas (quienes realizan el proceso de metanogénesis), protozoarios y bacteriófagos, los cuales han coevolucionado con los hospederos durante millones de años, volviéndose vitales para desarrollar las necesidades metabólicas de ambas partes (Morgavi,Forano,Martin y Newbold, 2010).

Hay varios factores que pueden llegar a afectar la producción de metano pero el principal factor reportado es la alimentación del bovino (Manzano Cué, Caraballoso Johnson \& Castro Carrillo, 2007), por lo que muchas de las estrategias para disminuir la cantidad de metano producido responden a la alimentación, en la cual se busca una alimentación de buena calidad que disminuya la metanogénesis.

La liberación de metano causada por una mala alimentación no solo llega a afectar al medio ambiente por la liberación de gases de efecto invernadero sino que también se ve afectado el metabolismo de los rumiantes disminuyendo su productividad y su utilización efectiva de energía (Kim y Gadd, 2008).

La cuantificación del metano permite estudiar el impacto de dichos factores en la metanogénesis producida por microorganismos en rumiantes (Goopy,Chang y Tomkins,2016). Existen dos formas de abordar la producción de metano; mediante el aislamiento de microorganismos anaeróbicos presentes en el rumen o con el intercambio gaseoso del rumiante. El primero presenta la ventaja de que no es invasivo, pero tiene la desventaja de que representa una muestra del total del metano producido (Liu,Yang,Ye y Han, 2018). Por otro lado, el segundo método mencionado mide directamente el metano liberado, pero depende estrictamente del comportamiento del rumiante y le puede generar un estrés adicional que sesgue los resultados (Storm et al., 2012).

Debido a los efectos negativos que representan la generación y liberación de metano al medio ambiente, se han desarrollado numerosas investigaciones en los últimos años en las cuales se proponen posibles estrategias de mitigación. Estas se pueden orientar hacia la disminución de la producción de los gases de efecto invernadero o al aumento de los mecanismos de captura de los compuestos formadores de dichos gases (Alayón-Gamboa et al., 2018). Dichas estrategias pueden ser clasificadas además en tres tipos: manipulación alimentaria y dietética, manipulación animal y manipulación microbiana. Las técnicas incluidas en estas influyen sobre el estado del ecosistema presente en el rumen y la tasa de metanogénesis (Pinares-Patiño, 2014). 
Con esta revisión se pretende compilar información existente sobre el proceso metanogénico de los rumiantes y los factores que lo afectan, las repercusiones y métodos de cuantificación del metano así como las diferentes medidas de mitigación que se han realizado.

\section{Microorganismos del rumen y producción del metano}

La metanogénesis es de particular importancia en los rumiantes, ya que estos liberan el $97 \%$ del metano que es producido por animales domésticos (Díaz, Castañeda, Endara, 2015). Las emisiones procedentes de la ganadería de rumiantes son entonces una fuente importante de metano, solamente en China esta actividad agropecuaria ocasiona el 7,2 \% de la producción global de este gas de efecto invernadero (Bonilla y Lemus, 2012). Conocer la diversidad de microorganismos que realizan el proceso es de suma importancia a la hora de realizar cambios en la microbiota ruminal. Se sabe que esta microbiota no permanece estática, sino que varía dependiendo de la dieta, una dieta rica en fibra provoca un aumento de aproximadamente el $27 \%$ en la diversidad de metanógenos del rumen comparado a una dieta rica en almidón en vacas lecheras analizadas en Latinoamérica (Belanche et al., 2012).

La vía metanogénica consiste en una variante anaerobia de la respiración celular en donde se oxida el hidrógeno molecular y se reduce el dióxido de carbono a metano (metanogénesis hidrogenotrófica), como se observa en la ecuación [1].

$$
4 \mathrm{H}_{2}+\mathrm{CO}_{2} \rightarrow \mathrm{CH}_{4}+2 \mathrm{H}_{2} \mathrm{O}
$$

Además del dióxido de carbono, el acetato (metanogénesis acetotrófica, ecuación [2] y compuestos metilados pueden funcionar como sustratos del proceso, pero aún así, el hidrógeno molecular reduce principalmente el $\mathrm{CO}_{2}$ (Ramírez, Ochoa, Noguera, 2014).

$$
\mathrm{CH}_{3} \mathrm{COO}^{-}+\mathrm{H}^{+} \rightarrow \mathrm{CH}_{4}+\mathrm{CO}_{2}
$$

Cuando se analiza la variante acetotrófica, es importante considerar su contraparte acetogénica, proceso en el que se sintetiza ácido acético a partir de dióxido de carbono e hidrógeno diatómico ecuación [3].

$$
2 \mathrm{CO}_{2}+4 \mathrm{H}_{2} \rightarrow \mathrm{CH}_{3} \mathrm{COOH}+2 \mathrm{H}_{2} \mathrm{O}
$$

Además del acetato, el propionato es otro ácido graso de cadena corta que funciona como intermediario, su oxidación por parte de bacterias libera hidrógeno molecular utilizado como sustrato de la metanogénesis (Yamada, Kato, Ueno, Ishii, Igarashi, 2014).

El proceso metanogénico es realizado por organismos del dominio Archaea (Lyu, Shao, Akinyemi \& Whithman, 2018). La modificación controlada del microbioma ruminal regula los niveles de metano liberados al ambiente. Se han estudiado los efectos del suministro de residuos agrícolas (rastrojo de maíz, semilla de algodón y colza) en los niveles de metano y se ha encontrado una disminución en estos, ya que generalmente tienen una cantidad elevada de taninos que puede 
modificar la riqueza de especies metanogénicas en el rumen, influenciando así los niveles de metano (Wang et al., 2016). Como menciona (Zhou y Hernández-Sanabria, 2009), la alimentación eficiente e ineficiente hace que la variabilidad en la composición de metanógenos sea menor y mayor respectivamente en términos de las unidades taxonómicas operacionales (técnica utilizada para clasificar organismos emparentados).

\section{Factores influyentes en la metanogénesis}

La metanogénesis es un proceso natural del metabolismo de los rumiantes, el cual no se puede eliminar, pero puede disminuirse su impacto, mediante estrategias alimenticias, dentro de las más utilizadas están: incrementos del consumo animal, modificación en la composición de la dieta, eliminación de los protozoos y mejora de la eficiencia de digestión de la fibra (Morgavi et al., 2010). Se ha demostrado que la tasa de emisión de metano por fermentación ruminal está relacionada con la especie, la edad y las características fisicoquímicas del alimento consumido por el ganado, lo que afecta directamente al nivel de consumo, lo que produce mayor cantidad de metano en el rumen cuando el animal consume forrajes poco digestibles y de baja calidad (Manzano Cué, Caraballoso Johnson \& Castro Carrillo, 2007).

La alimentación tradicional del ganado bovino de engorde consta de forrajes enteros los cuales proporcionan todos los nutrimentos esenciales, como por ejemplo la avena, alfalfa y otros, lo que genera altas emisiones de metano con efectos negativos sobre la productividad animal y la salud ambiental (Herrera,Jordany Senra,2010). Se ha demostrado que el uso de árboles forrajeros atenúan los efectos negativos. Los árboles forrajeros utilizados deben tener un valor nutricional elevado para que puedan utilizarse como un suplemento que reduzca el metano producido por la dieta a base de forraje de mala calidad los cuales no incluyen todos los compuestos necesarios para la dieta del rumiante. Estos árboles mantienen altos niveles de proteínas y minerales durante el crecimiento (Clavero, 2011).

Se han desarrollado técnicas para reducir el impacto ambiental de los rumiantes, las cuales constan de usar una mezcla de plantas con alto contenido de metabolitos secundarios (principalmente taninos) en la alimentación de los rumiantes reducen las emisiones de metano sin alterar la fermentación ruminal (Blanco, 2017). Se ha encontrado que en el caso de las ovejas al adicionarles $25 \mathrm{~g}$ de taninos de Acasia mearnssi / Kg de materia seca a la dieta, se reducen significativamente los niveles de metano emitido un $13 \%$ en promedio (Carulla,Vereuzer,Machmüller y Hess,2005). En el caso de las vacas lecheras de pastoreo, los niveles de metano disminuyeron un 32\% usando 120 gramos al día de extracto de taninos de $A$. mearnssi, aunque también ocasionan que la tasa de consumo de concentrado disminuya (Alves, Dall-Orsoletta y Ribeiro-Filho, 2017). 
Hay que observar los efectos colaterales que los taninos agregados a la dieta puedan generar. Por ejemplo, se ha encontrado que los extractos de $A$. mearnssillegan a afectar en el plazo de tres días la composición lipídica de la leche en vacas (Denninger et al., 2020). Un estudio realizado por Grainger et al. (2009) mostró que los taninos de $A$. mearnssiusados en un 0,9\% de la materia seca ingerida disminuyeron el metano producido en $14 \%$, pero los niveles de producción de leche bajaron un 3,6\% en promedio. Se ha tratado de emplear otras especies vegetales para tratar de disminuir el proceso metanogénico. Beauchemin,McGinn,Martinez y McAllister (2017) en su estudio utilizaron los taninos del árbol de quebracho, aunque no lograron reducir los niveles de metano, sin bien las concentraciones empleadas fueron bajas (2\% de la materia seca consumida) con el fin de disminuir el riesgo de intoxicación.

También se propone la elaboración de bloques multinutricionales a base de subproductos del cacao debido a su alto contenido en fibra (González, 2013); siendo un factor importante debido a que la fermentación de las paredes celulares de la fibra resulta en una alta proporción ácido acético: ácido propiónico que favorece la pérdida de $\mathrm{CH}_{4}$ (Bonilla y Lemus, 2012). Además, se sugiere utilizar leguminosas ya que optimizan el uso de la energía en los rumiantes disminuyendo la producción de metano en un 27 a un 31\% (Nuñez, 2019).

Se ha demostrado que la adición de ácidos grasos poliinsaturados de cadena larga disminuye la metanogénesis porque se convierte en una alternativa metabólica para el hidrógeno. Sin embargo, la cantidad total de hidrógeno usado en los procesos de biohidrogenación de los ácidos grasos insaturados endógenos es pequeña (1\%) comparado con el que es usado para la reducción de CO2 a metano (48\%), la síntesis de AGV (33\%) y la síntesis bacteriana (12\%) (Carmona, 2005).

Durante los últimos cinco años se ha producido abundante literatura relacionada con la proposición de alternativas de reducción de las emisiones de metano entérico por animal. Dichas propuestas de mitigación pueden clasificarse en tres categorías de manipulación, las cuales no son mutuamente excluyentes: manipulación alimentaria y dietética, animal y microbiana (Cuadro 1). En referencia a las anteriores, cualquier método de reducción de las emisiones de metano operaría a través de la alteración del ecosistema ruminal y la tasa de metanogénesis (PinaresPatiño, 2014). 
Cuadro 1. Categorías de manipulación propuestas para la reducción de emisiones de metano entérico.

\begin{tabular}{|c|c|c|c|}
\hline $\begin{array}{l}\text { Categoría de } \\
\text { Manipulación }\end{array}$ & Impacto Positivo & Impacto Negativo & Valor de Respuesta \\
\hline $\begin{array}{l}\text { Alimenticia } \\
\text { Dietética }\end{array}$ & $\begin{array}{l}\text { Aumenta la } \\
\text { productividad animal y } \\
\text { disminuye el rendimiento } \\
\text { de metano por unidad de } \\
\text { consumo. }\end{array}$ & $\begin{array}{l}\text { Se requiere un } \\
\text { mayor consumo } \\
\text { de alimento. }\end{array}$ & $\begin{array}{l}\text { Se requiere forraje de } \\
\text { buena calidad. } \\
\text { En sistemas intensivos } \\
\text { de producción se } \\
\text { recomiendan aditivos } \\
\text { dietéticos }\end{array}$ \\
\hline Microbial & $\begin{array}{l}\text { Se dirige el balance } \\
\text { de hidrógeno en beneficio } \\
\text { del animal. } \\
\text { Se puede reducir } \\
\text { directa e indirectamente } \\
\text { las poblaciones de } \\
\text { metanógenos. }\end{array}$ & $\begin{array}{l}\text { El control } \\
\text { de los } \\
\text { metanógenos } \\
\text { puede afectar } \\
\text { también a otros } \\
\text { microorganismos } \\
\text { del rumen. }\end{array}$ & $\begin{array}{l}\text { El microbioma del } \\
\text { rumen es difícil de } \\
\text { manipular. } \\
\text { Esta técnica aún se } \\
\text { encuentra en estudios } \\
\text { primarios de desarrollo. } \\
\text { Se ocupan dietas } \\
\text { controladas para mejorar } \\
\text { los efectos. }\end{array}$ \\
\hline Animal & $\begin{array}{l}\text { Eficiencia alimentaria es } \\
\text { heredable. } \\
\text { Se utilizan animales de } \\
\text { menor consumo residual } \\
\text { por lo que se gasta menos } \\
\text { alimento. }\end{array}$ & $\begin{array}{l}\quad \text { La selección } \\
\text { animal puede } \\
\text { comprometer las } \\
\text { características } \\
\text { funcionales del } \\
\text { animal. }\end{array}$ & $\begin{array}{l}\text { No hay aún una relación } \\
\text { clara entre el consumo } \\
\text { residual y las emisiones de } \\
\text { metano. } \\
\text { Se deben mejorar los } \\
\text { indicadores de consumo } \\
\text { residual para. }\end{array}$ \\
\hline
\end{tabular}

Fuente: Piñares-Patiño (2014)

\section{Emisiones de metano en rumiantes}

El $\mathrm{CH}_{4}$ proveniente de esta fermentación entérica representa el 30\% del total de emisiones atribuidas a la industria agropecuaria (Shibata y Terada, 2010). En el caso de los bovinos, son una de las principales fuentes antropogénicas de emisión de $\mathrm{CH}_{4}$, reportando datos de emisión de entre 300 a 600 l/CH4/animal /día (Cardona, Mahecha y Ángulo, 2017). 
En Costa Rica la producción ganadera tiene un alto valor económico y constantemente se incrementa el número de individuos y las áreas destinadas a la misma (Iñamagua-Uyaguari et al., 2016). Este sector para el año 2005 determinó un 39\% del total de emisiones de GEI correspondientes al metano (Chacón, Jiménez, Montenegro, Sasa y Blanco, 2015). Estos datos a lo largo de los años han traído un gran desequilibrio medio ambiental y una pérdida creciente en el valor productivo de la industria en el país; estudios recientes realizados en Costa Rica en la industria lechera, evidencian que las más vacas robustas y productoras emiten menos metano por kilogramo de leche producida, lo que demuestra que es posible desarrollar un enfoque de mitigación mediante la productividad, cubriendo la creciente demanda de productos derivados y los requerimientos ambientales ligados al cambio climático (Ballestero, Guevara y Cruz, 2020).

\section{Repercusiones de la metanogénesis en rumiantes}

La liberación de metano producto del metabolismo microbiano en animales rumiantes trae consigo diversas repercusiones tanto en el metabolismo y productividad comercial de los mismos como en la estabilidad del medio ambiente en general (Ramírez et al; 2014).

A nivel metabólico se da un proceso de fermentación en la digestión de los animales rumiantes y algunos productos de este proceso fermentativo como el dióxido de carbono y el hidrógeno, no son utilizados por el animal, pero sirven como sustrato para la comunidad de microorganismos metanógenos en su sistema los cuales producen $\mathrm{CH}_{4}$ como estrategia para obtener la energía necesaria para su crecimiento (Kim y Gadd, 2008). El proceso de metanogénesis cumple con un papel de aceptor de electrones removiendo continuamente el H2 del medio ruminal. Así, la formación de $\mathrm{CH}_{4}$ se torna esencial para el óptimo desempeño del ecosistema en el rumen, ya que evita la acumulación de $\mathrm{H}_{2}$ en el mismo, lo cual podría generar la inhibición de la actividad deshidrogenasa relacionada en la re-oxidación de los cofactores reducidos como NAD+; además, esta actividad metanogénica contribuye a la pérdida de carbono para el animal y consecuentemente la pérdida de energía afectando su desempeño y la productividad comercial (Martín, Rojas, Arenas y Herrera, 2017).

En términos ambientales el calentamiento global afecta la sostenibilidad ambiental y genera cambios drásticos en las temperaturas del planeta (Ortiz, 2012). El $\mathrm{CH}_{4}$ es el segundo gas de efecto invernadero de mayor relevancia y el proceso de metanogénesis ha contribuido ampliamente y de manera progresiva al aumento de sus concentraciones en la atmósfera debido que las emisiones de este gas producto de la metanogénesis entérica representa uno de los valores más altos a nivel mundial en la contribución de GEI (Cerón, Scorcione y Nieto, 2018). 


\section{Estrategias de mitigación de efectos metanogénicos}

La mitigación de los GEl en la ganadería se basa en la identificación de las diferentes fuentes emisoras en el sistema productivo. Una vez identificados, las estrategias o medidas de mitigación pueden orientarse hacia la disminución en la producción de dichos gases o el aumento de los mecanismos de captura (fuentes sumidero) de sus compuestos formadores (Alayón-Gamboa et al., 2018).

\section{Manipulación alimentaria y dieta}

Existe una relación directamente proporcional entre el nivel del consumo de materia seca y la producción de metano (Gerber, Henderson y Makkar, 2013). Sin embargo, el rendimiento de metano por unidad de consumo se reduce y la alimentación en abundancia resulta en una mayor productividad animal. A mayor consumo, menor tiempo de retención de la ingesta, por lo que la actividad celulolítica decrece y genera una menor tasa de digestión de fibra (el componente más metanogénico del alimento). Además, se favorece la fermentación productora de ácido propiónico en vez de ácido acético (Varón, 2015). Por otro lado, las dietas altas en concentrados (sobre todo aquellas con deficiencias en fibra) producen una disminución del pH ruminal, al cual los organismos metanogénicos son muy sensibles (Piñares-Patiño, 2014).

De esta forma, la composición nutricional de la dieta también influye de forma importante en la producción de metano. La presencia de fibra insoluble en las paredes celulares, presente en el forraje, favorece una mayor relación de acetato:propionato y una mayor producción de metano. Por otro lado, la fermentación de carbohidratos solubles produce menor producción de metano (Ungerfeld, Escobar-Bahamondes y Muñoz, 2018).

\section{Manipulación microbial}

Algunas de las técnicas investigadas como parte de la manipulación microbial comprenden el desarrollo de vacunas, desarrollo de la capacidad de fagocitosis, control de protozoarios ruminales, potenciación de los homoacetogénicos, desarrollo de inhibidores de metanogénesis y modificadores de fermentación. Las anteriores se caracterizan por tener el potencial para reducir de forma directa e indirecta la población y actividad de los metanogénicos ruminales, además de dirigir el balance de hidrógeno resultante en beneficio del animal, mediante la producción de ácido acético (Piñares-Patiño, 2014).

Una de las prácticas más representativas referentes a la manipulación microbial es la defaunación, la cual consiste en la eliminación de protozoos. Este grupo actúa de manera simbiótica con organismos metanógenos y estos producen una proporción importante del metano ruminal. Sin embargo, aún no existe un método de defaunación práctico a nivel productivo, aunque algunos 
extractos vegetales pueden disminuir el número de protozoos (Newbold, De la Fuente, Belanche, Ramos-Morales y McEwan , 2015; Orozco-Durán et al., 2016 y Ungerfeld et al., 2018).

\section{Manipulación animal}

Consiste básicamente en la selección artificial basada en la variabilidad inter-animal de individuos que contribuyan a una reducción en la emisión de GEI. Se opta por la crianza de animales con un bajo consumo neto de alimentos. Este consiste en la diferencia entre el consumo real del animal durante el periodo de medición y el consumo esperado basado en el peso del animal y su ganancia de peso, utilizando ecuaciones de balance energético. Los animales que consumen menos de lo esperado tienen un valor negativo de consumo neto, por lo que son más eficientes (Della Rosa, 2018). A pesar de que la relación entre el consumo neto de alimentos y las emisiones de $\mathrm{CH}_{4}$ no es todavía clara, para un mismo nivel de producción bovina se gastará menos alimento, por lo que se producirán menos emisiones de GEI si se utilizan animales de consumo residual negativo. No obstante, los efectos del mejoramiento de la productividad animal sobre las emisiones de los GEI solamente serán efectivos si se reduce el número de animales, de forma paralela (Piñares-Patiño, 2014).

En lo que a las unidades de producción ganadera respecta, existe una amplia gama de tecnologías basadas en buenas prácticas y agroforestería, con el fin de mitigar las emisiones de GEI. Una de las estrategias más prometedoras en cuanto a factibilidad de adopción para pequeños productores consiste en los sistemas silvopastoriles. Esta permite a su vez progresar hacia un modelo de ganadería orgánica con bajos insumos. El uso de árboles a su vez permite su aprovechamiento en forraje, leña, alimento, medicina, sombra, fertilización del suelo (por microorganismos fijadores de nitrógeno asociados) y la reducción de la deforestación al usar la vegetación arbórea en sucesión (Alayón-Gamboa et al., 2018).

\section{Métodos de cuantificación del metano}

La cromatografía de gases es una de las técnicas tradicionales por su alta precisión, antiinterferencia y adaptabilidad. Consiste en extraer una muestra de biogás, colocarla en el inyector y separarla en la columna. Mediante este proceso, se puede detectar el metano y calcular su porcentaje de concentración (Liu et al., 2018).

En los últimos años se ha intentado desarrollar métodos que requieren un menor presupuesto y que se pueden usar en sistemas de producción a menor escala. Taubner y Rittman (2016) propusieron una técnica de cuantificación indirecta del rango de producción volumétrica de metano mediante la medición del rango de producción volumétrica de agua. El proceso inicia con el cultivo de metanógenos en lotes de cultivos cerrados, como frascos de suero. El principio 
del método se basa en la ecuación [1], a partir de cual se puede cuantificar la producción de $\mathrm{CH}_{4}$ mediante la reducción obtenida en la presión dentro del frasco de suero y valorar el incremento en la masa líquida por la producción de $\mathrm{H}_{2} \mathrm{O}$. La principal ventaja de este nuevo método es que permite cuantificar cultivos puros de metanógenos respecto a su rango de evolución de metano volumétrico (Taubner y Rittman, 2016). Fernández, Gomis-Tena, Hernández y Saiz (2019) desarrollaron un sistema calorimétrico indirecto a circuito abierto para rumiantes pequeños. Presenta una ventaja respecto a los métodos anteriores dado que se puede aplicar directamente al rumiante en estudio y también presenta alta repetibilidad. El estudio consiste en medir mediante un equipo de calorimetría el intercambio gaseoso asociado con la oxidación de sustratos energéticos $y$, con base en las diferencias de concentraciones entre aspiración y expiración del individuo, determinar el calor producido. A pesar de su reducido costo respecto a las cámaras de respiración y a su precisión del 99\%, aun presenta el reto de ser independiente a la conducta del individuo.

\section{CONSIDERACIONES FINALES}

La metanogénesis ruminal es ocasionada por arqueas que como parte su metabolismo produce metano a partir de diferentes sustratos orgánicos y depende de factores como la alimentación y la diversidad de organismos metanogénicos presentes en el rumen.

El metano es generado como consecuencia de la fermentación microbiana de la materia orgánica presente en el alimento. El animal representa sólo un hospedero, el cual influye de forma indirecta en la tasa de metanogénesis a través de su dieta, tasa de consumo, tamaño ruminal, masticación/salivación y tasa de pasaje.

La implementación de medidas de mitigación de la metanogénesis es necesaria debido a la rápida afectación que sus gases generan al incrementar el calentamiento global y al valor productivo de los animales. Sin embargo, al incorporar dichas medidas en sistemas de producción se debe hacer una evaluación para asegurarse que al reducir los niveles de metano no se afecten también los estándares de productividad del animal (niveles y composición de leche como se mencionó anteriormente). Además, se debe promover el desarrollo de un modelo productivo económicamente viable con un reducido impacto ambiental, con el fin de que este sea sostenible.

La revisión realizada muestra que existe una abundante experimentación con la alimentación del ganado como estrategia para la manipulación de la microbiota. Unir estos esfuerzos con métodos de detección de metano, como el caso mencionado anteriormente de la calorimetría en las vacas podrían ayudar a establecer un perfil adecuado para los animales rumiantes que se trabajan en el país. Lo anterior se puede adicionalmente unir a los esfuerzos por disminuir los gases de efecto invernadero, lo cual caracteriza el plan de desarrollo del país hacia una economía más amigable con el medio ambiente. 


\section{LITERATURA CITADA}

Alayón-Gamboa, J. A., Jiménez-Ferrer, G., Piñeiro-Vázquez, Á. T., Canul-Solís, J., Albores-Moreno, S., Villanueva-López, G. \& Ku-Vera, J. C. (2018). Estrategias de mitigación de gases de efecto invernadero en la ganadería. Agroproductividad, 11(2).

Alves, T. P., Dall-Orsoletta, A. C., \& Ribeiro-Filho, H. M. N. (2017). The effects of supplementing Acacia mearnsii tannin extract on dairy cow dry matter intake, milk production, and methane emission in a tropical pasture. Tropical animal health and production, 49(8), 1663-1668.

Ballestero, J. M., Guevara, E. B., \& Cruz, S. I. (2020). Cuantificación de metano entérico según estado fisiológico en vacas lecheras de alta producción en Costa Rica. Agronomía Costarricense, 44(1), 79-92.

Beauchemin, K. A., McGinn, S. M., Martinez, T. F., \& McAllister, T. A. (2007). Use of condensed tannin extract from quebracho trees to reduce methane emissions from cattle. Journal of Animal Science, 85(8), 1990-1996.

Belanche, A., Doreau, M., Edwards, J. E., Moorby, J. M., Pinloche, E., \& Newbold, C. J. (2012). Shifts in the rumen microbiota due to the type of carbohydrate and level of protein ingested by dairy cattle are associated with changes in rumen fermentation. The Journal of nutrition, 142(9), 1684-1692.

Blanco Fontao, C. (2017). Desarrollo de nuevos sistemas de alimentación y estrategias para la producción de carne de ovino (Tesis doctoral). Universidad de León, España.

Bonilla Cárdenas, J. A., \& Lemus Flores, C. (2012). Emisión de metano entérico por rumiantes y su contribución al calentamiento global y al cambio climático: Revisión. Revista mexicana de ciencias pecuarias, 3(2), 215-246.

Cardona-Iglesias, J. L., Mahecha-Ledesma, L., \& Angulo-Arizala, J. (2017). Fodder shrubs and fatty acids: strategies to reduce enteric methane production in cattle [Arbustos forrajeros y ácidos grasos: estrategias para reducir la producción de metano entérico en el ganado]. Agronomía Mesoamericana, 28(1), 273-288.

Carmona, J., Bolívar, D., \& Giraldo, L. (2005). El gas metano en la producción ganadera y alternativas para medir sus emisiones y aminorar su impacto a nivel ambiental y productivo. Revista Colombiana de Ciencias Pecuarias, 18(1), 49-63.

Carulla, J. E., Kreuzer, M., Machmüller, A., \& Hess, H. D. (2005) Supplementation of Acacia mearnsii tannins decreases methanogenesis and urinary nitrogen in forage-fed sheep. Australian journal of agricultural research, 56 (9), 961-970. 
Cerón Cucchi, M. E., Scorcione Turcato, M. C., \& Nieto, M. I. (2018). Alternativas para la reducción de la emisión de metano en bovinos. XLI REUNIÓN CIENTÍFICA ANUAL-2018, 55.

Chacón Araya, A. R., Jiménez Valverde, G., Montenegro Ballestero, J., Sasa Marín, J., \& Blanco Salas, K. (2015). Inventario nacional de gases de efecto invernadero y absorción de carbono, 2012 (No. Formato digital). Ministerio de Ambiente y Energía, San José (Costa Rica). Instituto Meteorológico Nacional.

Clavero, T. (2011). Agroforestería en la alimentación de rumiantes en América Tropical. RU Zulia, 2(2), 11-35.

Denninger, T. M., Schwarm, A., Birkinshaw, A., Terranova, M., Dohme-Meier, F., Münger, A.,\& Kreuzer, M. (2020). Immediate effect of Acacia mearnsii tannins on methane emissions and milk fatty acid profiles of dairy cows.Animal Feed Science and Technology, 261, 114388.

Díaz, B., Castañeda, S., \& Endara, G. (2015). Aporte a la mitigación ambiental mediante el uso de microorganismos para reducir la producción de metano en rumiantes. Perfiles, 13 (1), 18.

Della Rosa, M. M. (2018). Variaciones en la calidad de la carne asociadas al consumo residual de bovinos en pastoreo (Doctoral dissertation, Facultad de Ciencias Agrarias, Universidad Nacional de Mar del Plata).

Fernández, C., Gomis-Tena, J., Hernández, A., \& Saiz, J. (2019). An Open-Circuit Indirect Calorimetry Head Hood System for Measuring Methane Emission and Energy Metabolism in Small Ruminants. Animals, 9(6), 380.

Galindo, J., Elías, A., Muñoz, E., Marrero, Y., González, N., \& Sosa, A. (2017). Ruminal activators, general features and their advantages for feeding ruminants. Revista Cubana de Ciencia Agrícola, 51 (1), 11-23.

Gerber, P. J., Henderson, B., \& Makkar, H. P. (2013). Activadores ruminales, aspectos generales y sus ventajas en la alimentación de animales rumiantes. Cuban Journal of Agricultural Science. 51 (1), p 11-23.

Goopy J.P., Chang C. \& Tomkins N. (2016). A comparison of methodologies for measuring methane emissions from ruminants. In: Rosenstock T., Rufino M., Butterbach-Bahl K., Wollenberg L., Richards M. (eds) Methods for Measuring Greenhouse Gas Balances and Evaluating Mitigation Options in Smallholder Agriculture. Springer, Cham.

González, B. (2013). Efecto en la fermentación ruminal in vitro de dietas utilizadas para ganado lechero, adicionadas con especies altas en taninos. (Tesis de maestría, Universidad Autónoma del Estado de México). 
Grainger, C., Clarke, T., Auldist, M. J., Beauchemin, K. A., McGinn, S. M., Waghorn, G. C., \& Eckard, R. J. (2009). Potential use of Acacia mearnsii condensed tannins to reduce methane emissions and nitrogen excretion from grazing dairy cows. Canadian Journal of Animal Science, 89(2), 241-251.

Herrera, J., Jordán, H., \& Senra, A. F. (2010). Aspectos del manejo y alimentación de la reproductora ovina Pelibuey en Cuba. Revista Cubana de Ciencia Agrícola, 44(3), 211-219.

Iñamagua-Uyaguari, J. P., Jenet, A., Alarcón-Guerra, L. G., Vilchez-Mendoza, S. J., Casasola-Coto, F., \& Wattiaux, M. A. (2016). Impactos económicos y ambientales de las estrategias de alimentación en lecherías de Costa Rica. Agronomía Mesoamericana, 27(1), 1-17.

Kim, B.H., \& Gadd G.M. (2008). Bacterial physiology and metabolism. Cambridge, Inglaterra: Cambridge University Press.

Liu, X., Yang, J., Ye, T., \& Han, Z. (2018). Establishment of analysis method for methane detection by gas chromatography. IOP Conf. Series: Earth and Environmental Science (Vol. 113, p.012023).

Lyu, Z., Shao, N., Akinyemi, T., \& Whitman, W. B. (2018). Methanogenesis. Current Biology, 28(13), 727-732.

Manzano Cué, A., Caraballoso Johnson, A., \& Castro Carrillo, M. (2007). Emisión de metano en la cría de búfalo, alternativas para aminorar su impacto ambiental en la UBPC la 5 del proyecto sabana camagüey.

Martín, Y., Rojas, G., Arenas, N., \& Herrera, V. (2017). Alternativas nutricionales para disminuir emisiones de gas metano por bovinos y su efecto en el calentamiento global. Revista Ciencias Agropecuarias, 3 (1), 6-23

Morgavi, D. P., Forano, E., Martin, C., \& Newbold, C. J. (2010). Microbial ecosystem and methanogenesis in ruminants. Animal: an international journal of animal bioscience, 4(7), 1024.

Newbold, C. J., De La Fuente, G., Belanche, A., Ramos-Morales, E., \& McEwan, N. R. (2015). The role of ciliate protozoa in the rumen. Frontiers in microbiology, 6, 1313.

Núñez-Torres, O. P., \& Rodríguez-Barros, M. A. (2019). Subproductos agrícolas, una alternativa en la alimentación de rumiantes ante el cambio climático. Journal of the Selva Andina Animal Science, 6(1), 24-37.

Orozco-Durán, K. E., Herrera-Camacho, J., Castelán-Ortega, O. A., Márquez-Benavides, L., Buenrostro-Delgado, O., \& Kú-Vera, J. C. (2016). Reducción de la metanogénesis ruminal in vitro con aceites vegetales de Thevetia peruviana y Persea americana. Ecosistemas y recursos agropecuarios, 3(9), 335-344. 
Ortiz, R. (2012). El cambio climático y la producción agrícola. Banco Interamericano de Desarrollo, p13-17.

Pinares-Patiño, C. (2014). Mitigación de las emisiones de metano entérico de sistemas ganaderos al pastoreo. In Primera conferencia de gases de efecto invernadero en sistemas agropecuarios de Latinoamérica (GALA). Serie de Actas (No. 54, pp. 25-31).

Ramírez, J. F., Ochoa, S. P., \& Noguera, R. (2014). Metanogénesis ruminal y estrategias para su mitigación. Revista CES Medicina Veterinaria y Zootecnia, 9(2), 307-324.

Shibata, M., \& Terada, F. (2010). Factors affecting methane production and mitigation in ruminants. Animal Science Journal, 81(1), 2-10.

Taubner, R. S., \& Rittmann, S. K. M. (2016). Method for indirect quantification of CH4 production via $\mathrm{H} 2 \mathrm{O}$ production using hydrogenotrophic methanogens. Frontiers in microbiology, 7 , 532.

Ungerfeld, E. M., Escobar-Bahamondes, P., \& Muñoz, C. (2018). Predicción y mitigación de las emisiones de metano de los rumiantes. AGROProductividad, 11(2), 34-40.

Varón, L. E. S. (2015). Las dietas en las emisiones de metano durante el proceso de rumia en sistemas de producción bovina. Revista de Investigación Agraria y Ambiental (RIAA), 2(1), 55-64.

Wang, P., Zhao, S., Wang, X., Zhang, Y., Zheng, N., \& Wang, J. (2016). Ruminal methanogen community in dairy cows fed agricultural residues of corn stover, rapeseed, and cottonseed meals. Journal of agricultural and food chemistry, 64(27), 5439-5445.

Yamada, C., Kato, S., Ueno, Y., Ishii, M., \& Igarashi, Y. (2014). Conductive iron oxides accelerate thermophilic methanogenesis from acetate and propionate. Journal of Bioscience and Bioengineering. ND (N.D), 1-5

Zhou, M. I., \& Hernandez-Sanabria, E. (2009). Assessment of the microbial ecology of ruminal methanogens in cattle with different feed efficiencies. Applied and environmental microbiology, 75(20), 6524-6533. 